patients to be either Duke criteria negative, or Duke possible and not treated for IE. PWID referred to the IE team who were either Duke definite or Duke possible and treated for IE acted as a comparison group. Patient demographics, microbiology, final diagnoses, survival and causes of death were recorded.

Results There were 112 episodes of other infections in 107 PWID during the study period. 69 (65\%) patients were male, and the mean age was $36 \pm 8.4$ years. During the same period, we assessed and treated 105 episodes of confirmed IE in 92 PWID. There were positive blood cultures in 71 episodes, most commonly Staphylococcus aureus (39), coagulase negative Staphylococcus (3), beta-haemolytic Streptococcus (16), oral Streptococcus (7), Streptococcus angionosis (4), Enterococcus spp. (2), Pseudomonad (1) and other (7). In 10 episodes there was combined infection with two or more microorganisms, and in 41 episodes blood cultures were negative. Where IE was excluded, the final diagnosis was usually an infected deep vein thrombosis (DVT) (41) or skin and soft tissue infection (26). Other infections included osteomyelitis (9), septic arthritis (6), septic pulmonary emboli/lung abscess (5), central line infection (4), pneumonia (4), urinary tract infection (2), pericarditis (2) and in one episode there was a cerebral abscess. Survival following hospitalisation with an episode of infection was $91 \%, 86.4 \%, 83.5 \%$ and $70 \%$ at $1,3,5$ and 10 years respectively. In univariate analysis, survival was significantly higher in PWID with other infections compared to those treated for IE during the same period $(p=0.0002)$ (figure 1). During a median follow-up of 4.8 years there were a total of 26 deaths, with suicide or drug overdose being the cause of death in 9 cases.

Conclusion In approximately half of PWID referred to the IE team the final diagnosis was non-cardiac infection. In those with other infections the alternative diagnosis was usually an infected DVT or skin/soft tissue infection. Survival was favourable in those with other infections compared to those treated for IE. The implication of this finding is that cardiac infection has a negative impact on survival in PWID beyond infection elsewhere. It seems likely that damaged native heart valves, or indwelling prosthetic material poses additional risk of re-infection in the context of ongoing drug use. The continuing high mortality rate in both groups suggests that improving the support offered to PWID might affect outcomes in the longer term.

Conflict of Interest None

\section{DIAGNOSIS OF ARRHYTHMOGENIC CARDIOMYOPATHY AND OVERLAP WITH CARDIAC ADAPTATION TO EXERCISE: INSIGHTS FROM A CARDIAC MAGNETIC RESONANCE STUDY}

${ }^{1}$ Gherardo Finocchiaro*, ${ }^{2}$ Eleonora Moccia, ${ }^{3}$ Stathis Papatheodorou, ${ }^{4}$ Chris Miles, ${ }^{3}$ Abbas Zaidi, ${ }^{3}$ Elijah Behr, ${ }^{3}$ Nicholas Bunce, ${ }^{3}$ Lisa Anderson, ${ }^{4}$ Sanjay Sharma, ${ }^{3}$ Ahmed Merghani, ${ }^{4}$ Michael Papadakis. 'Guy's and St Thomas' Hospital; ${ }^{2}$ Universita' degli Studi di Sassari; ${ }^{3}$ St George's University Hospital; ${ }^{4}$ St George's University of London

\subsection{6/heartjnl-2019-BCS.128}

Background The diagnosis of arrhythmogenic right ventricular cardiomyopathy (ARVC) is often challenging and structural abnormalities typical of the disease may overlap with changes reflective of cardiac adaptation to exercise. The aim of the study was to assess the performance of the cardiac magnetic resonance (CMR) 2010 Task Force Criteria (TFC) in a cohort of patients with definite diagnosis of ARVC and define the overlap with a cohort of highly trained athletes of similar age and sex.

Methods We compared the CMR features of 43 patients (mean age $49 \pm 17$ years, 49\% males, 32 (74\%) genotyped) with a definite diagnosis of ARVC according to the revised TFC to 97 (mean age $45 \pm 16$ years, 61\% males) highly-trained athletes of similar age and sex, where cardiac disease was excluded after comprehensive work-up.

Results The CMR was abnormal in 37 (86\%) patients. The $\mathrm{RV}$ was affected in isolation in 17 (39\%) patients, with 18

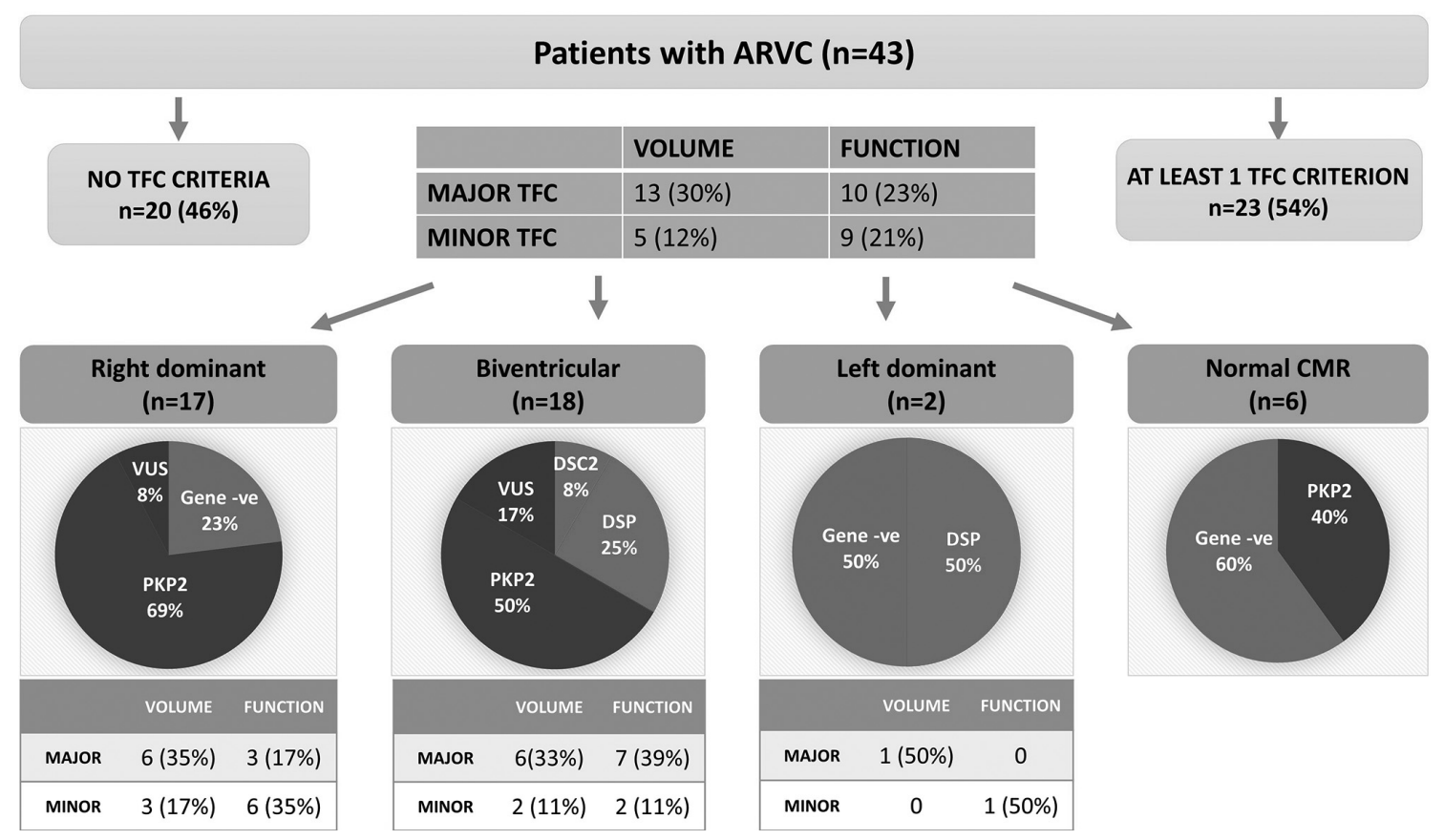

Abstract 131 Figure 1 
(42\%) patients exhibiting biventricular involvement and 2 $(5 \%)$ patients showing isolated left ventricular involvement. The most common RV abnormalities were regional wall motion abnormalities (RWMA) $(\mathrm{n}=34 ; 79 \%)$, RV dilatation fulfilling a major or minor volume TFC $(n=18 ; 42 \%)$, impaired systolic function (RVEF $\leq 45 \%: n=17 ; 40 \%$ ) and myocardial fibrosis $(n=13 ; 30 \%)$. The predominant LV abnormality was myocardial fibrosis $(n=20 ; 47 \%)$, with a small proportion of patients exhibiting RWMA $(n=6 ; 14 \%)$ and impaired systolic function (LVEF <50\%: $n=6 ; 14 \%$ ).

Twenty-three $(53 \%)$ patients fulfilled a major $(n=14 ; 32 \%)$ or a minor $(n=9 ; 21 \%)$ CMR TFC. Sixteen $(16 \%)$ athletes exceeded the cut-off values for RV volumes used as a major $(n=10 ; 10 \%)$ or a minor $(n=6 ; 6 \%)$ TFC with an inverse relationship between age and RV volumes $(r=-0.41, p=0.001)$. None of the athletes fulfilled the TFC for RV ejection fraction. Applying the CMR TFC to the ARVC patient population, showed a sensitivity of $33 \%$ for the major and $28 \%$ for the minor criteria. Applying the RV volume and systolic function TFC values in the entire study population, showed a sensitivity of $53 \%$, a specificity between $83 \%$ and an accuracy of 0.68. Please see (Figure 1).

Conclusion The great majority (86\%) of patients with ARVC demonstrate structural abnormalities suggestive of cardiomyopathy on CMR but only $53 \%$ fulfil any of the CMR TFC. A small proportion $(16 \%)$ of older athletes demonstrate significant RV dilatation that overlaps with the volume criteria for ARVC. The emergence of ARVC as a biventricular disease provides an opportunity to re-evaluate the diagnostic criteria and include LV involvement in conjunction with RV involvement to improve diagnostic accuracy.

Conflict of Interest no

\section{THE ADDITIONAL VALUE OF CARDIOPULMONARY EXERCISE TESTING OVER CONVENTIONAL TREADMILL TESTING IN PATIENTS WITH MODERATE TO SEVERE AORTIC STENOSIS}

${ }^{1}$ Sveeta Badiani*, ${ }^{2}$ Jet van Zalen, ${ }^{2}$ Lesley Hart, ${ }^{2}$ Ann Topham, ${ }^{1}$ Karen Armado, ${ }^{1}$ Ricardo Monteiro, 'David Hoare, ${ }^{1}$ Aeshah Althunayyan, ${ }^{1}$ Sahar Borikan, ${ }^{2}$ Andrew Marshall, ${ }^{11}$ Sanjeev Bhattacharyya, ${ }^{2}$ Nik R Patel, ${ }^{1}$ Guy Lloyd. ${ }^{1}$ Barts Health NHS Trust; ${ }^{2}$ Eastbourne District General Hospital

\subsection{6/heartjnl-2019-BCS.129}

Introduction Current guidelines recommend aortic valve replacement in patients with apparently asymptomatic severe aortic stenosis, who exhibit symptoms or a decrease in blood pressure on exercise testing. However, the additional information provided by cardiopulmonary exercise testing (CPET) is limited. This study sought to establish the additional information provided by CPET in this context.

Methods An analysis of patients with aortic stenosis undergoing CPET in an enhanced valve surveillance clinic was performed. Symptom limited cardiopulmonary exercise testing with respiratory gas exchange analysis was used, and the patients were encouraged to exercise until exhaustion.

Results 71 patients with moderate to severe aortic stenosis and good left ventricular function (AVVmax $4 \quad 0.5 \mathrm{~m} / \mathrm{s} \quad \mathrm{MG} 38$ $11 \mathrm{mmHg}$, AVA $0.90 .2 \mathrm{~cm}^{2}$, LVEF $608 \%$ )were included in the analysis. The mean age was $74.2 \quad 12.1$ years and 51 patients $(70.4 \%)$ were male. $33(46.5 \%)$ patients had a background of hypertension, $12(16.9 \%)$ had diabetes mellitus and $8(11.3 \%)$ had coronary artery disease.

All patients denied cardiovascular symptoms at baseline. A total of 119 CPETs were performed. All patients exercised to respiratory exchange ratio (RER) $>1.1$ indicating good effort.

\section{Abstract 132 Table 1}

\begin{tabular}{|c|c|}
\hline Baseline demographics & \\
\hline Age (years) & $74.2 \pm 12.1$ \\
\hline Gender (male) & $51(70.4 \%)$ \\
\hline Hypertension & $33(45.8 \%)$ \\
\hline Diabetes mellitus & $12(16.9 \%)$ \\
\hline Coronary artery disease & $8(11.3 \%)$ \\
\hline Height $(\mathrm{cm})$ & $169 \pm 9$ \\
\hline Weight $(\mathrm{kg})$ & $77 \pm 14$ \\
\hline \multicolumn{2}{|l|}{ Echocardiographic data } \\
\hline Peak aortic velocity $(\mathrm{m} / \mathrm{s})$ & $4 \pm 0.5$ \\
\hline Mean gradient $(\mathrm{mmHg})$ & $38 \pm 11$ \\
\hline Aortic valve area $\left(\mathrm{cm}^{2}\right)$ & $0.9 \pm 0.2$ \\
\hline Left ventricular ejection fraction (\%) & $60 \pm 8$ \\
\hline \multicolumn{2}{|l|}{ Cardiopulmonary exercise testing data } \\
\hline Power (watts) & $91 \pm 49$ \\
\hline Resting heart rate (bpm) & $73 \pm 12$ \\
\hline Peak heart rate $(\mathrm{bpm})$ & $123 \pm 18$ \\
\hline Resting systolic blood pressure (mmHg) & $149 \pm 22$ \\
\hline Peak systolic blood pressure (mmHg) & $176 \pm 25$ \\
\hline Peak VO2 (ml/min/kg) & $18 \pm 5.2(88 \pm 16 \%$ predicted $)$ \\
\hline VE/VCO2 slope & $30.9 \pm 6.5$ \\
\hline Oxygen uptake efficiency slope & $1787 \pm 599(97 \pm 26 \%$ predicted $)$ \\
\hline Oxygen pulse & $11 \pm 3.1(103 \pm 26 \%$ predicted $)$ \\
\hline
\end{tabular}

